Revista Signos 2009, 42(71) $385-408$

\title{
Metonimias ilocutivas y categorización de peticiones y órdenes en el español de Chile*
}

\author{
Claudia Muñoz \\ Universidad de Concepción \\ Chile
}

Resumen: En este artículo presentamos los resultados de dos pruebas de prototipicidad, cuyo objetivo fue obtener evidencia de que las diferencias en la representatividad de enunciados indirectos de petición y de orden dependen tanto de la centralidad de los componentes de los respectivos 'modelos cognitivos' que son instanciados como del grado en que están presentes en los enunciados. El trabajo se sustenta en el enfoque metonímico delineado por Thornburg y Panther (1997), el que ha sido ampliado por Pérez Hernández y Ruiz de Mendoza (2002). De acuerdo con este enfoque, los actos de habla indirectos son tipos de 'metonimia', es decir, enunciados capaces de evocar un determinado 'Modelo Cognitivo Idealizado Ilocutivo', a través de la expresión de uno de sus componentes. Los resultados de las pruebas verifican la dependencia esperada y pueden ser explicados desde la perspectiva metonímica.

Palabras Clave: Actos de habla, metonimia, categorización, petición, orden.

*Este artículo se desarrolló con el apoyo de la Dirección de Investigación de la Universidad
de Concepción, Proyecto DIUC 206.063.006-1.0, 2007-2009.

Recibido: 27-VI-2008 Aceptado: 3-III-2009
Correspondencia: Claudia Muñoz (claumuno@udec.cl). Departamento de Filosofía, Universidad de Concepción. Los Olmos 1280, Barrio Universitario, Concepción, Chile. 


\title{
Illocutionary metonymies and categorization of requests and commands in Chilean Spanish
}

\begin{abstract}
This paper presents the results from two prototypicality tests which aimed at obtaining evidence to prove that the differences in the representativity of indirect request and command utterances depend on both the centrality of the components of the respective cognitive models that are instantiated and the degree to which they are present in the utterances. This work is based on Thornburg and Panther's (1997) metonymic approach, which has been dealt with in depth by Pérez Hernández and Ruiz de Mendoza (2002). According to this approach, indirect speech acts are types of 'metonymy', that is, utterances that can evoke a particular 'Illocutionary Idealized Cognitive Model' through the expression of one of its components. The findings confirm the expected dependence and can be explained from the metonymyc point of view.
\end{abstract}

Key Words: Speech acts, metonymy, categorization, request, command.

\section{INTRODUCCIÓN}

En el amplio campo de estudios abierto por la Teoría de los Actos de Habla, gran parte de la preocupación de los teóricos se ha centrado en el problema de la interpretación de la fuerza ilocutiva indirecta. En la tradición inferencialista (Searle, 1975; Grice, 1975; Leech, 1997; Sperber \& Wilson, 1994) las respuestas ofrecidas suelen partir de una división más o menos estricta entre semántica (significado de la oración) y pragmática (intención comunicativa). La tarea del analista, especialmente en la tradición convencionalista de la pragmática inferencial, consiste en dar cuenta de la clase de estrategias interpretativas (inferencias) que son necesarias para calcular la intención del hablante a partir del 'acto de habla literal'. En Lingüística Cognitiva, Panther y Thornburg (1998) y Pérez Hernández y Ruiz de Mendoza (2002), quienes han tratado el tema y realizado los desarrollos más importantes y novedosos en esta línea de trabajo, coinciden en que el enfoque inferencialista tradicional no es adecuado, por dos razones principales: primero, porque no resulta coherente con la rapidez interpretativa y la gran diversidad expresiva cotidiana; segundo, porque no ofrece una descripción sistemática de los patrones inferenciales y de su 'anclaje' (grounding) cognitivo.

En este trabajo seguimos, en gran parte, la propuesta de Thornburg y Panther (1997), según la cual el significado ilocutivo se halla organizado, en el conocimiento de las personas, en la forma de 'Escenarios de Actos de Habla' (Speech Act Scenarios; Thornburg \& Panther, 1997). Sobre la base semántica representada por estos escenarios, opera la 'metonimia', que es concebida como un instrumento para la realización indirecta de la fuerza pragmática (Thornburg \& Panther, 1997), y como guía del trabajo inferencial. Aplicaremos el enfoque metonímico en el análisis de un grupo de enunciados directivos cuya prototipicidad para las categorías de la 
petición y de la orden fue medida en dos pruebas de categorización aplicadas en 2008 a un grupo de 43 estudiantes de tercer año de enseñanza media del Colegio Bautista de Concepción, de Chile. Dichos enunciados representan metonimias de los componentes 'ANTES' (BEFORE), 'NÚCLEO' (CORE) y 'DESPUÉS' (AFTER) del escenario de directivos de Thornburg y Panther (1997). Las pruebas nos permitieron obtener evidencia de que el potencial ilocutivo directivo (índice de prototipicidad) de expresiones novedosas (no literales), es decir, no convencionalizadas (en el sentido de Ruiz de Mendoza, 2001) para la expresión de la fuerza directiva de petición y de orden, depende del potencial metonímico de los componentes representados y del grado en que los mismos están presentes en ellas, lo que nos permite afirmar también que las metonimias ilocutivas de atributos relevantes favorecen la percepción de centralidad categorial de las expresiones. La relevancia de los componentes del escenario de directivos debe entenderse con respecto a la medida (óptima o mínima) en que se encuentran representados cognitivamente. Derivamos nuestras hipótesis (o sub-hipótesis) de uno de los principios hipotéticos formulados por Thornburg y Panther (1997), referido a la probabilidad de que un determinado componente del escenario de directivos esté en una relación metonímica PARTE-TODO con el escenario.

Se debe aclarar que nuestro propósito no fue la detección de eventuales prototipos de petición y de orden, sino la obtención de un ordenamiento de los enunciados ('efecto de gradiencia'), con respecto a estas categorías, que pudiera explicarse a partir del enfoque metonímico.

\section{Marco teórico}

Panther y Thornburg (2007) señalan el importante rol que cumple la metonimia en la organización del significado (semántica), en la producción e interpretación de enunciados (pragmática) e incluso en la estructura gramatical. "Los mismos principios metonímicos que relacionan diferentes sentidos de una palabra sirven para crear y recuperar significados novedosos en el uso efectivo del lenguaje" (Panther \& Thornburg, 2007: 236). Así, por ejemplo, los principios metonímicos EFECTO POR CAUSA y POTENCIALIDAD POR ACTUALIDAD, operan en la construcción de enunciados metonímicos de la misma manera que las metáforas conceptuales subyacen a la productividad metafórica. Según esto, la metonimia no sería solamente una figura del habla ni tendría tan solo la función referencial que le atribuye la tradición, sino que se encontraría, también, en el nivel conceptual. Su amplio y relevante rol, destacan Panther y Thornburg (2007), es un indicio de que la división estricta entre semántica y pragmática, como componentes que actúan autónomamente, no es adecuada. Los numerosos trabajos en Lingüística Cognitiva sobre la participación de principios metonímicos y su interacción con modelos metafóricos y esquemas de imagen, en las diversas dimensiones del uso del lenguaje, fortalecen la conclusión de que no habría tal separación, sino más bien "un continuum entre el significado lingüístico (semántica) y el uso comunicativo (pragmática)” (Panther \& Thornburg, 2007: 236). La idea es que mucho de 
nuestro conocimiento acerca de las necesidades, las intenciones y los objetivos de los participantes en los procesos de comunicación se encuentran sistematizados en estructuras cognitivas que sirven de guía en la labor inferencial (Ruiz de Mendoza, 2001; Pérez Hernández, 2000).

\subsection{Metonimias ilocutivas}

En el campo de los estudios sobre actos de habla, Thornburg y Panther (1997) han propuesto que la realización (e interpretación) de acciones lingüísticas indirectas puede ser entendida como el resultado de la participación de principios metonímicos. La ventaja de este enfoque es que se condice, mejor que las propuestas inferencialistas tradicionales, con la automaticidad y rapidez interpretativa cotidiana, y también con la diversidad expresiva de la fuerza pragmática. La naturaleza convencional y operacional de la metonimia supone una disminución en el costo de procesamiento, ya que hace innecesario postular procesos intermedios de interpretación -como el de la identificación de la fuerza ilocutiva literal y su comparación con el contexto comunicativo- y permite su uso para regular la actividad inferencial (Pérez Hernández \& Ruiz de Mendoza, 2002).

Una 'metonimia ilocutiva' (o 'metonimia de acto de habla') es un caso en el que un componente de un 'escenario ilocutivo', que es lo suficientemente 'central', puede "evocar metonímicamente otros componentes del escenario y, de este modo, el escenario como un todo" (Panther \& Thornburg, 2007: 247). La idea básica es que un atributo de un acto de habla puede 'estar por' el acto de habla, del mismo modo en que un atributo de una persona puede estar por la persona.

Un 'Escenario de Acto de Habla' es un tipo de Modelo Cognitivo Idealizado $(\mathrm{MCl})$ que surge de la generalización de situaciones directivas típicas, como órdenes, peticiones, consejos, sugerencias, etc. (Pérez Hernández \& Ruiz de Mendoza, 2002). Tiene una estructura proposicional secuencial de tres fases o etapas: el ANTES (BEFORE), el NÚCLEO y el RESULTADO (CORE, RESULT), y el DESPUÉS (AFTER). Estas fases incluyen las condiciones de fortuna descritas por Searle (1969, 1975): 'preparatorias', 'esenciales', 'de sinceridad' y 'proposicionales', y cada una puede 'estar por' el escenario como un todo en la expresión de un acto de habla.

El componente ANTES contiene las condiciones pragmáticas y discursivas que permiten que se realice la acción lingüística. Por ejemplo, la 'Habilidad del oyente $(0)$ de realizar la acción (A)' es una precondición de los actos de habla directivos, mientras que la 'Actitud emocional del hablante $(\mathrm{H})$ con respecto a un hecho p' es una condición de los expresivos.

La próxima fase, dadas las precondiciones del componente ANTES, corresponde al aspecto central del acto de habla, al que Searle (1969) denominara 'condición esencial'. Este se divide en dos subfases: el NÚCLEO y sus RESULTADOS, que contienen, respectivamente, las propiedades 
que definen el acto lingüístico y las consecuencias directas o inmediatas de su realización exitosa; por ejemplo, en el escenario directivo de la orden, el NÚCLEO se define por el intento del hablante de 'poner a $\mathrm{O}$ bajo la obligación fuerte de hacer A'; y si la orden es exitosa, entonces el RESULTADO es que ' $O$ queda bajo la obligación fuerte de hacer $A$ '.

La tercera fase del escenario, el DESPUÉS, incluye las consecuencias pragmáticas, intentadas o no intentadas, del acto de habla, pero que no son su resultado inmediato. Tales consecuencias se refieren a la realización del contenido proposicional; por ejemplo, que 'O realizará A', en el caso de los actos directivos, o que 'H realizará A', en los actos de habla compromisivos.

El siguiente es el escenario de actos de habla directivos de Thornburg y Panther (1997: 208):

\section{Escenario para Actos de Habla Directivos}

(1) ANTES: 1.0 puede hacer A.

2. $\mathrm{H}$ quiere que $O$ haga $A$.

(2) NÚCLEO: $\quad \mathrm{H}$ pone a $\mathrm{O}$ bajo una obligación (más o menos fuerte) de hacer $\mathrm{A}$.

RESULTADO: 0 está bajo una obligación de hacer $\mathrm{A}$ ( $\mathrm{O}$ debe / debería / debiera hacer $\mathrm{A}$ ).

(3) DESPUÉS: 0 hará $A$.

Los ejemplos (1), (2) y (3) son, respectivamente, metonimias de los componentes ANTES, NÚCLEO y DESPUÉS del escenario de directivos; es decir, casos en los que la fuerza ilocutiva directiva se expresa a través de la referencia a alguno de estos componentes, el que está, en cada caso, en una relación metonímica ('estar por') con el escenario.

(1) ¿Puedes acercarme esa caja? (ANTES)

(2) Tienes que salir de aquí (RESULTADO)

(3) Te acostarás temprano hoy (DESPUÉS)

La forma en que un atributo del acto de habla 'está por' el acto de habla mismo (PARTE POR EL TODO) puede explicarse "reduciendo las metonimias de acto de habla a principios metonímicos más generales" (Panther \& Thornburg, 1999: 344). La metonimia ilocutiva en el ejemplo (1) es un subcaso del principio metonímico más general POTENCIALIDAD POR ACTUALIDAD; mientras que las metonimias en los ejemplos (2) y (3) son subcasos de la metonimia EL EFECTO POR LA CAUSA. Estos principios subyacen, entonces, a la producción de las metonimias ilocutivas ejemplos (1), (2) y (3) y son, también, los patrones que guían su interpretación. Se supone, por lo tanto, que "los hablantes tienen almacenados los escenarios ilocutivos en su memoria a largo 
plazo" (Pérez Hernández \& Ruiz de Mendoza, 2002: 262), los que pueden ser evocados metonímicamente por las expresiones de actos de habla. Dado que las condiciones expresivas se basan en principios metonímicos, permiten a los hablantes trazar las inferencias necesarias para calcular, con gran rapidez, la intención ilocutiva del emisor.

\subsection{Prototipo y modelos cognitivos idealizados}

La lingüística cognitiva ha desarrollado la discusión sobre los 'conceptos' o 'categorías' en términos de las nociones de 'prototipo' y 'parecido familiar', conformando así un conjunto de conocimientos y métodos de experimentación conocido como Semántica de Prototipos o Teoría del Prototipo. Los desarrollos iniciales provienen de las investigaciones llevadas a cabo por Rosch (1973a, 1973b, 1975), Labov (1973) y Rosch y Mervis (1975) acerca del fenómeno de la 'categorización', cuyos resultados son coincidentes en gran medida con los estudios de Berlin y Kay (1969) sobre los 'términos básicos de color' (basic color terms) y los 'colores focales'; sus antecedentes filosóficos, por otra parte, se encuentran en las ideas de 'parecidos de familia' (Familienähnlichkeiten) y 'juegos de lenguaje' (Sprachspiele), introducidas por Ludwig Wittgenstein (1953) en su crítica de la teoría clásica o esencialista del significado.

En este trabajo usamos la noción de prototipo categorial para referirnos a un complejo de propiedades representativas entendido dentro del marco más amplio que representan los $\mathrm{MCls}$, descritos por Lakoff (1987). Lakoff desarrolla su propuesta sobre MCls a partir de cuatro fuentes: la 'semántica de marcos' de Fillmore (1982), la teoría de la metáfora y la metonimia de Lakoff y Johnson (1995), la noción de 'esquemas de imagen' de la gramática cognitiva de Langacker (1986) y la teoría de los 'espacios mentales' de Fauconnier (1985). La 'semántica de marcos' de Fillmore (1982) es similar en muchos sentidos a la teoría del 'esquema' de Rumelhart (1975) y de los scripts de Schank y Abelson (1977). Un MCl es un conjunto de propiedades altamente idealizadas, o un complejo de varios modelos cognitivos, cada uno entendido como un conjunto de propiedades, que puede estructurarse por principios proposicionales (Fillmore, 1982), esquemáticos (Langacker, 1986), metonímicos o metafóricos (Lakoff \& Jonson, 1980). Según Lakoff (1987), tanto la estructura de las categorías como los efectos de prototipo dependen de la existencia de los $\mathrm{MCls}$. Siguiendo este enfoque, entendemos los actos verbales como tipos de categorías de prototipo, es decir, estructuras conceptuales de límites difusos, definidas a partir de ciertos $\mathrm{MCls}$ ilocutivos y que producen efectos de prototipicidad, especialmente el de gradiencia. Este efecto no depende de la categoría de prototipo en sí misma, sino del $\mathrm{MCl}$ ilocutivo correspondiente, definido aquí como un complejo de propiedades que pueden ser realizadas en diversos grados por las emisiones (Pérez Hernández, 2000). Según esto, no es necesaria la presencia de todas las propiedades del $\mathrm{MCl}$, en los contextos correspondientes y en los enunciados, para que la emisión sea clasificada como una expresión de esa categoría. Coleman y Kay (1981) 
ofrecen evidencia a favor de esta idea. A través de su prueba de categorización ellos encontraron que para la caracterización del significado del verbo lie los sujetos no parecían tener condiciones necesarias y suficientes, y sus respuestas se fundaban más bien en un 'conjunto' de tres condiciones: a) 'falsedad', b) 'falta de creencia' del hablante y c) 'intento de engañar,' ninguna de las cuales era necesaria y todas presentaban variados grados de importancia.

Los MCls reúnen en sí, u organizan de manera simplificada, información convencional sobre aspectos de la realidad (eventos, situaciones, contextos, etc.), pero no siempre se acomodan perfectamente a la experiencia real o concreta que las personas tienen de esa realidad, sino que aparecen a menudo desprendidos de esa experiencia y pueden incluso sobreponerse a ella, formatearla (Rivano, 1999). Es esta idealización o fuerte limitación de los MCls la fuente de los efectos de prototipicidad, incluso en casos de categorías clásicas (Lakoff, 1987). Los MCls constituyen así esquemas o patrones recurrentes y significativos de la regularidad de nuestra experiencia (Johnson, 1987). Los actos de habla pueden entenderse también como MCls de tipo proposicional que intervienen tanto en la producción de actos verbales como en la interpretación de la fuerza o intención comunicativa. Pérez Hernández y Ruiz de Mendoza (2002) proponen que en la conceptualización de los actos de habla directivos participan modelos de 'esquemas de imagen' en el sentido de Johnson (1987). En nuestro trabajo no desarrollaremos este aspecto de su propuesta, pero sí mostraremos ejemplos cuyo grado de representatividad para las categorías de orden y petición puede explicarse por la interacción de los $\mathrm{MCls}$ escénicos con 'esquemas de distancia conceptual' (Taylor, 2003), para producir distintos niveles de obligatoriedad y de opcionalidad, idea que estos autores destacan también en el citado artículo de 2002.

\subsection{La hipótesis de Thornburg y Panther}

Según Thornburg y Panther (1997), no cualquier componente puede estar en una relación metonímica con el escenario. Consideremos, por ejemplo, las peticiones (4) y (5), que involucran la transferencia de objetos:

(4) ¿Tienes café?

(5) ¿Tienes café, por favor?

Su utilidad para realizar peticiones se basa en que la condición ' $O$ tiene (está en posesión de) el objeto X', que expresan, "se presupone de la condición más importante de que ' 0 pueda proporcionar X al hablante H'” (Thornburg \& Panther, 1997: 211), que equivale al componente ANTES 1 ('Habilidad de 0 de hacer A') del escenario de directivos. La relevancia de esta última condición, comparada con las presuposiciones que involucra (' $O$ tiene $X$ ' y ' $X$ existe'), aumen- 
ta, según Thornburg y Panther (1997), la probabilidad de que esté en una relación metonímica ('estar por') con el escenario correspondiente. Las presuposiciones de que ' $O$ tiene $X$ ' y de que ' $X$ existe' pueden, cuando son expresadas en enunciados como (4) y (5), 'apuntar al' escenario, pero es menos probable que 'estén por' el escenario. Esto explica que (4) necesite más marcadores de petición ('por favor') para contar como una expresión de la fuerza ilocutiva peticionaria, y que (5), que incorpora el marcador, exprese esta fuerza más claramente que (4). Asimismo, explica que (6) represente más claramente que (4) y (5) una instancia de petición, pues en (6) se destaca una condición relevante y fuente de las presuposiciones que (4) y (5) expresan.

\section{(6) ¿Puedes darme un café?}

Según Thornburg y Panther (1997), la centralidad de los componentes de un escenario de acto de habla y el número de estos que expresa un enunciado, permiten predecir el potencial metonímico del componente, así como la probabilidad de que ese enunciado sea interpretado como un ejemplo del acto de habla correspondiente. Esta hipótesis es desglosada por los autores en los siguientes principios (Thornburg \& Panther, 1997: 211):

(a) Mientras más localizado está un componente del acto de habla en la periferia del escenario correspondiente, con menos probabilidad este componente estará en una relación 'estar por' (metonímica) con el escenario.

(b) Mientras más componentes del escenario estén presentes en una pieza de discurso, más fácil será trazar inferencias de la parte al todo.

Interpretamos estas hipótesis (o principios) en términos de prototipicidad, es decir, como indicaciones de que la relevancia de los componentes, su grado de manifestación y el número de los mismos que está presente en un enunciado, permiten predecir el potencial ilocutivo del enunciado en cuestión, es decir, su poder para expresar, con independencia de un contexto específico, una determinada fuerza ilocutiva. Ruiz de Mendoza (2001) llama 'construcciones ilocutivas' a las oraciones cuyo contenido es lo suficientemente explícito para inducir al oyente a captar el sentido ilocutivo que se desea comunicar. Se trata, en cierta medida, de estructuras que presentan una alta coincidencia entre forma y función, pero que, primordialmente, instancian modelos cognitivos específicos. Esto explicaría que su sentido pueda interpretarse prescindiendo en gran medida del contexto de uso. La 'literalidad' aparece, en este caso, como un 'continuum construccional' en el que los diferentes enunciados ilocutivos pueden ubicarse de acuerdo a su grado de especialización para instanciar un modelo específico de acto de habla. A medida que disminuye su grado de especialización, aumenta también el grado de dependencia del contexto y, por tanto, la indirección ilocutiva. 


\section{Pruebas de prototipicidad}

Si consideramos contextos experimentales en los que los modelos ilocutivos específicos de petición y de orden sean activados para producir gradación, pueden plantearse las siguientes subhipótesis, en concordancia con el principio (a) de Thornburg y Panther (1997):

(a.1.) Si se presentan enunciados contexto-no-marcados que destaquen los cuatro componentes del escenario de Thornburg y Panther, y pedimos a un grupo de sujetos que establezcan en qué medida cada uno de ellos representa un ejemplo útil para realizar peticiones (Prueba A) y órdenes (Prueba B), podremos establecer, a partir de los índices de prototipicidad de los enunciados, qué componentes del escenario de actos de habla directivos, por su relevancia en las decisiones de categorización, tienen mayores y menores probabilidades de estar en una relación metonímica ('estar por') con los MCls de ambas clases de actos de habla.

(a.2) Si, además, en los enunciados que expresan un mismo componente ilocutivo hacemos variar el grado de expresión del mismo, podremos establecer relaciones de dependencia entre estas diferencias y las variaciones en sus índices de prototipicidad para peticiones (Prueba A) y órdenes (Prueba B).

Tomamos como referencia para nuestras pruebas y para la evaluación de los resultados, los $\mathrm{MCls}$ de Petición y de Orden propuestos por Pérez Hernández y Ruiz de Mendoza (2002). Estos incluyen el escenario de directivos de Thornburg y Panther (1997), más propiedades específicas que estos autores no consideraron, como vemos en las Figuras 1 y 2 :

Escenario de Panther y Thornburg más:

(i) A representa un costo para $\mathrm{O}$ y un beneficio para $\mathrm{H}$.

(ii) Alta opcionalidad (cortesía).

(iii) La relación de poder entre $\mathrm{H}$ y $\mathrm{O}$ es irrelevante.

Figura 1. Modelo Cognitivo Idealizado de Petición.

Escenario de Panther y Thornburg más:

(i) A representa un costo para $\mathrm{O}$ y un beneficio para $\mathrm{H}$.

(ii) Baja opcionalidad (ausencia de cortesía)

(iii) $\mathrm{H}$ es más poderoso que 0.

Figura 2. Modelo Cognitivo Idealizado de Orden. 
Según Pérez Hernández (2000), los casos concretos de orden (y de los actos de habla en general) se ordenarán desde prototípicos a periféricos según se acerquen o se alejen de los valores óptimos para cada uno de los parámetros del $\mathrm{MCl}$. Las órdenes prototípicas expresan los valores en su grado más elevado, a la vez que representan un mayor número de los mismos.

\subsection{Metodología}

Se aplicó una prueba de prototipicidad para peticiones (Prueba A) y otra para órdenes (Prueba B) a dos grupos diferentes de sujetos, todos alumnos de tercer año medio del Colegio Bautista de Concepción: el grupo de la Prueba A, compuesto por 23 sujetos, y el grupo de la Prueba B, integrado por 20. En ambas pruebas se presentaron los mismos 22 enunciados, que expresaban distintos componentes del escenario de directivos de Thornburg y Panther (1997). En el análisis solo se consideraron los enunciados indirectos, mientras que ejemplos de formatos altamente convencionalizados (como el interrogativo de la forma 'Puedes + SV') se incluyeron entre los ejemplos, únicamente como referentes para asegurar el valor descriptivo de la prueba, en la medida en que se esperaba que resultaran prototípicos de petición y marginales para orden. Replicando el experimento de Coleman y Kay (1981), los sujetos debían calificar cada enunciado, dentro de una escala de prototipicidad (Tabla 1), según el grado en que, en su opinión, correspondía a un ejemplo 'útil' para realizar una petición (Prueba A) o una orden (Prueba B).

A partir de las respuestas se obtuvo el 'índice de prototipicidad' de cada enunciado, equivalente a su 'puntuación promedio' en la prueba. Este índice corresponde al grado en que los sujetos, como grupo, consideraron que la expresión era un ejemplo útil para realizar el acto de habla. El enunciado se considera más prototípico si su puntuación promedio es más cercana al índice absoluto 7 y menos prototípico si su puntuación promedio es más cercana a valor mínimo 1.

Tabla 1. Escala de utilidad de los enunciados para ejecutar peticiones.

\begin{tabular}{|c|c|c|c|c|c|c|}
\hline \multicolumn{2}{|c|}{ NO es útil para hacer una petición } & $\begin{array}{c}\text { No puedo } \\
\text { decir SÍ/NO }\end{array}$ & \multicolumn{3}{c|}{ Sí es útil para hacer una petición } \\
\hline 1 & 2 & 3 & 4 & 5 & 6 & 7 \\
\hline $\begin{array}{c}\text { Totalmente } \\
\text { seguro }\end{array}$ & $\begin{array}{c}\text { Medianamente } \\
\text { seguro }\end{array}$ & $\begin{array}{c}\text { Levemente } \\
\text { seguro }\end{array}$ & $\begin{array}{c}\text { Cualquiera de } \\
\text { los dos }\end{array}$ & $\begin{array}{c}\text { Levemente } \\
\text { seguro }\end{array}$ & $\begin{array}{c}\text { Medianamente } \\
\text { seguro }\end{array}$ & $\begin{array}{c}\text { Totalmente } \\
\text { seguro }\end{array}$ \\
\hline
\end{tabular}

Para calcular la puntuación promedio (o índice de prototipicidad) por enunciado se obtuvo la 'puntuación total' de cada uno, y se dividió esta por el número total de sujetos de la prueba $(23 / 20)$. La 'puntuación total' por expresión se obtuvo de multiplicar cada valor (rango) de la 
escala de 1 a 7 por el número de veces que este valor fue seleccionado por los sujetos al evaluar el enunciado, y sumando los siete resultados obtenidos.

Los enunciados de las pruebas representaban funciones metonímicas de cada uno de los componentes del escenario para actos de habla directivos de Thornburg y Panther (1997):

(i) ANTES 1: Habilidad de 0 para realizar A.

ANTES 2: $\quad$ Motivación / Deseo de $\mathrm{H}$ de que $\mathrm{O}$ realice $\mathrm{A}$.

(ii) NÚCLEO: $\mathrm{H}$ pone a $\mathrm{O}$ bajo una obligación (más o menos fuerte) de hacer $\mathrm{A} / \mathrm{O}$ está bajo una obligación (más o menos fuerte) de hacer A.

(iii) DESPUÉs: 0 hará A

El componente ANTES fue dividido en dos subcomponentes: ANTES 1 y ANTES 2; mientras que el componente NÚCLEO-RESULTADO fue considerado como una unidad, que llamamos solo NÚCLEO. Esta reducción se debió a la dificultad para formular 'metonimias de NÚCLEO' propiamente tales, es decir, enunciados que expresaran el 'intento de poner a 0 bajo una obligación de hacer $A^{\prime}$. Los ejemplos de la prueba destacan el resultado ilocutivo, más que la intención ilocutiva. Además, en los enunciados que representaban la misma metonimia de acto de habla, se hizo variar el modo de presentación del componente ilocutivo con el objeto de representar diversos grados de expresión del mismo (por ejemplo, ‘Podrías cerrar la puerta?’ y 'Si pudieras cerrar la puerta').

\subsection{Resultados}

Los resultados de las pruebas de prototipicidad de peticiones y órdenes se presentan en las Tablas 2 y 3, respectivamente. En las secciones siguientes, se analizan según sub-hipótesis y por componente directivo. 
Tabla 2. Prueba A.

\begin{tabular}{|l|c|}
\hline \multicolumn{1}{|c|}{ ENUNCIADOS-PETICIÓN } & ÍNDICE \\
\hline ¿Puedes cerrar la puerta? & 6,52 \\
\hline ¿Podrías cerrar la puerta? & 5,77 \\
\hline ¿Cerrarías la puerta? & 5,57 \\
\hline Quisiera que cierres la puerta. & 4,26 \\
\hline Querría que cierres la puerta. & 3,87 \\
\hline Necesitaría que cierres la puerta. & 3,83 \\
\hline Tú cierras la puerta, ¿ya? & 3,26 \\
\hline Si pudieras cerrar la puerta. & 3,26 \\
\hline Necesito que cierres la puerta. & 3,13 \\
\hline Si puedes cerrar la puerta. & 3,09 \\
\hline ¿No puedes cerrar la puerta? & 3,00 \\
\hline Deberías cerrar la puerta. & 2,96 \\
\hline Quiero que cierres la puerta. & 2,78 \\
\hline ¿Cerrarás la puerta? & 2,78 \\
\hline Tendrías que cerrar la puerta. & 2,30 \\
\hline Tienes que cerrar la puerta. & 2,26 \\
\hline Tú cerrarás la puerta. & 2,22 \\
\hline ¡Ya!. Me cerró la puerta. & 2,00 \\
\hline Debes cerrar la puerta. & 1,96 \\
\hline Vas a cerrar la puerta. & 1,91 \\
\hline Debieras cerrar la puerta. & 1,87 \\
\hline Si cerraras la puerta. & 1,83 \\
\hline
\end{tabular}

Tabla 3. Prueba B.

\begin{tabular}{|l|c|}
\hline \multicolumn{1}{|c|}{ ENUNCIADOS-ORDEN } & ÍNDICE \\
\hline Tienes que cerrar la puerta. & 6,40 \\
\hline Debes cerrar la puerta. & 6,05 \\
\hline Tú cerrarás la puerta. & 5,95 \\
\hline Vas a cerrar la puerta. & 5,40 \\
\hline Tú cierras la puerta, iya? & 5,05 \\
\hline Quiero que cierres la puerta. & 5,00 \\
\hline Necesitaría que cierres la puerta. & 4,95 \\
\hline Quisiera que cierres la puerta. & 4,70 \\
\hline Debieras cerrar la puerta. & 4,65 \\
\hline Deberías cerrar la puerta. & 4,40 \\
\hline Tendrías que cerrar la puerta. & 4,40 \\
\hline Necesito que cierres la puerta. & 4,30 \\
\hline Si cerraras la puerta. & 4,00 \\
\hline ¿Cerrarás la puerta? & 3,35 \\
\hline Querría que cierres la puerta. & 3,30 \\
\hline ¡Ya!. Me cerró la puerta. & 3,30 \\
\hline Si puedes cerrar la puerta. & 3,20 \\
\hline ¿No puedes cerrar la puerta? & 2,84 \\
\hline Si pudieras cerrar la puerta. & 2,79 \\
\hline ¿Podrías cerrar la puerta? & 2,05 \\
\hline ¿Puedes cerrar la puerta? & 1,90 \\
\hline ¿Cerrarías la puerta? & 1,75 \\
\hline
\end{tabular}

\subsubsection{Potencial metonímico de los componentes (Sub-hipótesis a.1.)}

De acuerdo con nuestra sub-hipótesis (a.1.), debíamos encontrar diferencias en el potencial de los componentes del escenario de directivos para relacionarse metonímicamente con los $\mathrm{MCls}$ de petición y de orden. Si un componente está en una relación metonímica con alguno de estos $\mathrm{MCls}$, el enunciado metonímico del caso debería ser altamente eficaz para realizar la fuerza ilocutiva asociada a ese modelo. En las siguientes tablas se presentan los resultados de prototipicidad de ambas pruebas, por componente. 
Tabla 4

\begin{tabular}{|l|c|}
\hline ANTES 1 - PETICIÓN & ÍNDICE \\
\hline ¿Puedes cerrar la puerta? & 6,52 \\
\hline ¿Podrías cerrar la puerta? & 5,77 \\
\hline Si pudieras cerrar la puerta. & 3,26 \\
\hline Si puedes cerrar la puerta. & 3,09 \\
\hline ¿No puedes cerrar la puerta? & 3,00 \\
\hline
\end{tabular}

Tabla 6

\begin{tabular}{|l|c|}
\hline ANTES 2 - PETICIÓN & ÍNDICE \\
\hline Quisiera que cierres la puerta. & 4,26 \\
\hline Querría que cierres la puerta. & 3,87 \\
\hline Necesitaría que cierres la puerta. & 3,83 \\
\hline Necesito que cierres la puerta. & 3,13 \\
\hline Quiero que cierres la puerta. & 2,78 \\
\hline
\end{tabular}

Tabla 8

\begin{tabular}{|l|c|}
\hline NÚCLEO - PETICIÓN & ÍNDICE \\
\hline Deberías cerrar la puerta. & 2,96 \\
\hline Tendrías que cerrar la puerta. & 2,30 \\
\hline Tienes que cerrar la puerta. & 2,26 \\
\hline Debes cerrar la puerta. & 1,96 \\
\hline Debieras cerrar la puerta. & 1,87 \\
\hline
\end{tabular}

Tabla 10

\begin{tabular}{|l|c|}
\hline DESPUÉS - PETICIÓN & ÍNDICE \\
\hline ¿Cerrarías la puerta? & 5,57 \\
\hline Tú cierras la puerta, ¿ya? & 3,26 \\
\hline ¿Cerrarás la puerta? & 2,78 \\
\hline Tú cerrarás la puerta. & 2,22 \\
\hline ¡Ya!. Me cerró la puerta. & 2,00 \\
\hline Vas a cerrar la puerta. & 1,91 \\
\hline Si cerraras la puerta. & 1,83 \\
\hline
\end{tabular}

Tabla 5

\begin{tabular}{|l|c|}
\hline ANTES 1 - ORDEN & ÍNDICE \\
\hline Si puedes cerrar la puerta. & 3,20 \\
\hline ¿No puedes cerrar la puerta? & 2,84 \\
\hline Si pudieras cerrar la puerta. & 2,79 \\
\hline ¿Podrías cerrar la puerta? & 2,05 \\
\hline ¿Puedes cerrar la puerta? & 1,90 \\
\hline
\end{tabular}

Tabla 7

\begin{tabular}{|l|c|}
\hline ANTES 2 - ORDEN & ÍNDICE \\
\hline Quiero que cierres la puerta. & 5,00 \\
\hline Necesitaría que cierres la puerta. & 4,95 \\
\hline Quisiera que cierres la puerta. & 4,70 \\
\hline Necesito que cierres la puerta. & 4,30 \\
\hline Querría que cierres la puerta. & 3,30 \\
\hline
\end{tabular}

Tabla 9

\begin{tabular}{|l|c|}
\hline NÚCLEO - ORDEN & ÍNDICE \\
\hline Tienes que cerrar la puerta. & 6,40 \\
\hline Debes cerrar la puerta. & 6,05 \\
\hline Debieras cerrar la puerta. & 4,65 \\
\hline Deberías cerrar la puerta. & 4,40 \\
\hline Tendrías que cerrar la puerta. & 4,40 \\
\hline
\end{tabular}

Tabla 11

\begin{tabular}{|l|c|}
\hline DESPUÉS - ORDEN & ÍNDICE \\
\hline Tú cerrarás la puerta. & 5,95 \\
\hline Vas a cerrar la puerta. & 5,40 \\
\hline Tú cierras la puerta, ¿ya? & 5,05 \\
\hline Si cerraras la puerta. & 4,00 \\
\hline ¿Cerrarás la puerta? & 3,35 \\
\hline ¡Ya!. Me cerró la puerta. & 3,30 \\
\hline ¿Cerrarías la puerta? & 1,75 \\
\hline
\end{tabular}

Como se observa en estas tablas, la expresión de diferentes fases del escenario de directivos en los enunciados se relaciona con diferencias en su grado de prototipicidad, para petición y orden, lo que nos permite hacer las predicciones que se presentan a continuación. 


\subsubsection{Componente ANTES}

El componente ANTES 1 ('Habilidad de 0 de hacer A') tiene mayores probabilidades de 'estar por' el $\mathrm{MCl}$ de la petición que el componente ANTES 2 ('Motivación/Deseo de $\mathrm{H}$ de que $\mathrm{O}$ haga $A^{\prime}$ '). A la vez, es más probable que el componente ANTES 2 'esté por' el $\mathrm{MCl}$ de la orden que por el de la petición. Entonces, las metonimias ilocutivas HABILIDAD DEL OYENTE POR PETICIÓN (ejemplos (7) y (8)) y MOTIVACIÓN DEL HABLANTE POR ORDEN (enunciados (9) y (10)) son muy probables.

(7) ¿Puedes cerrar la puerta?

(8) ¿Podrías cerrar la puerta?

(9) Quiero que cierres la puerta.

(10) Necesitaría que cierres la puerta.
(6,52 - petición / 1,90 - orden)

(5,77 - petición / 2,05 - orden)

(2,78 - petición / 5,00 - orden)

(3,83 - petición / 4,95 - orden)

Lo anterior supone que el componente ANTES 1 es más central para peticiones y que ANTES 2 es más central para órdenes, lo que puede explicarse porque la expresión de la 'Habilidad del oyente' (ANTES 1), al ejecutar un acto de habla directivo, supone mayor 'opcionalidad' de negarse a realizar la acción, mientras que la expresión del 'Deseo del hablante' (ANTES 2) centra el enunciado en el 'beneficio' del emisor del acto verbal, "disminuyendo la cortesía, o acrecentando la creencia descortés" (Leech, 1997: 177-182). La Tabla 4 muestra que los ejemplos (7) y (8), en que se pone en perspectiva el componente ANTES 1 de directivos, obtuvieron los índices de prototipicidad más altos para petición del grupo de 23 enunciados, mientras que los mismos obtuvieron índices bajos de prototipicidad para orden (Tabla 5). Asimismo, los enunciados (9) y (10), que destacan el componente ANTES 2, obtuvieron índices más altos para orden (Tabla 7), y más bajos para petición (Tabla 6).

\subsubsection{Componente NÚCLEO}

El componente NÚCLEO se encuentra altamente asociado al concepto de orden, por lo que nuestros resultados son esperables (Tablas 8 y 9): el componente NÚCLEO tiene más probabilidades de estar en una relación metonímica con el $\mathrm{MCl}$ de la orden y menos probabilidades de estar en una relación metonímica con el $\mathrm{MCl}$ de la petición. Lo que interesa destacar, sin embargo, es la información que nuestros datos ofrecen sobre las posibilidades de convencionalización ilocutiva de ciertos formatos (instancias ilocutivas potenciales), en relación con los principios metonímicos que operan. Un enunciado no literal para la realización de órdenes (de acuerdo con los parámetros pragmáticos tradicionales), que exprese una metonimia de NÚCLEO (ejemplos (11) al (15)), puede, activado el contexto ideal (MCI) de orden, producir respuestas adecuadas al modelo (en este caso, ser incluido en la categoría). Es posible esperar, entonces, que el uso 
de esta clase de enunciados metonímicos en situaciones reales produzca respuestas directivas automáticas (interpretación directiva y, eventualmente, la realización de la acción por parte de 0).

(11) Tienes que cerrar la puerta.

(12) Debes cerrar la puerta.

(13) Debieras cerrar la puerta

(14) Deberías cerrar la puerta

(15) Tendrías que cerrar la puerta
(2,26 - petición / 6,40 - orden)

(1,96 - petición / 6,05 - orden)

(1.87 - petición / 4.65 - orden)

(2.96 - petición / 4.40 - orden)

(2.30 - petición / 4.40 - orden)

\subsubsection{Componente DESPUÉS}

De los siete enunciados que expresaban el componente DESPUÉS de directivos, seis obtuvieron índices de mínima representatividad para petición (entre 3,26 y 1,83; ver Tabla 10); mientras que cuatro obtuvieron índices altos para orden (entre 5,95 y 4,00; ver Tabla 11). Por ejemplo, los enunciados (16) al (18), que destacan la consecuencia pragmática de que 'O hará A', obtuvieron índices de representatividad mayores para orden y menores para petición. De acuerdo con estos resultados, es más probable que el componente DESPUÉS 'esté por' el modelo de la orden, y menos probable que 'esté por' el modelo de la petición (Tablas 10 y 11). Lo que nos permite predecir que la probabilidad de la metonimia ilocutiva ACCIÓN FUTURA DEL OYENTE POR ORDEN es mucho mayor que la probabilidad de la metonimia ACCIÓN FUTURA DEL OYENTE POR PETICIÓN.

(16) Tú cerrarás la puerta

(17) Vas a cerrar la puerta

(18) Tú cierras la puerta ¿ya?

(19) ¿Cerrarías la puerta?
(2,22 - petición / 5,95 - orden)

(1,91 - petición / 5,40 - orden)

(3,26 - petición / 5,05 - orden)

(5, 57 - petición / 1,75 - orden)

Sin embargo, el ejemplo (19) obtuvo un alto índice de representatividad en la Prueba A, y es claramente un caso de la última metonimia. Creemos, pese a esto, que es posible mantener nuestra conclusión de que la probabilidad de la metonimia en cuestión es menor. Suponemos que el componente DESPUÉS es directivamente central, porque se expresa en su grado óptimo en directivos fuertes, como la orden. Si el grado de expresión es bajo, como en (19), aumenta el nivel de potencialidad o virtualidad de la 'acción futura del oyente', lo que motiva índices más altos de prototipicidad para categorías directivas menos fuertes, como la petición. Volveremos sobre esto en la sección 2.2.2.3., a propósito de la sub-hipótesis (a.2.). 


\subsubsection{Grados de expresión de los componentes (Sub-hipótesis a.2.)}

Las conclusiones acerca de la alta o baja probabilidad de que un determinado componente del escenario de directivos esté en una relación metonímica con los $\mathrm{MCls}$ de orden y petición, no son aplicables a todos los resultados obtenidos en nuestras pruebas de prototipicidad. Particularmente, no siempre la expresión de un mismo componente fue asociada exclusivamente, y con elevados índices, a la misma categoría (casos especiales son las metonimias de DESPUÉS y de NÚCLEO), lo que confirma nuestra sub-hipótesis (a.2.). Según esta, debíamos encontrar relaciones de dependencia entre las variaciones en los índices de prototipicidad (para petición y orden) de los enunciados que destacan un mismo componente ilocutivo y las diferencias en el grado de expresión de este componente en esos enunciados. A continuación exponemos las conclusiones con respecto a esta sub-hipótesis, para cada componente.

\subsubsection{Componente ANTES}

De acuerdo con nuestras conclusiones en 2.2.1.1., existe una alta probabilidad de que el componente ANTES 1 ('Habilidad de O') esté en una relación metonímica con el $\mathrm{MCl}$ de la petición, y de que el componente ANTES 2 ('Motivación/Deseo de $\mathrm{H}$ de que $\mathrm{O}$ haga A') lo esté con el $\mathrm{MCl}$ de la orden. Decíamos que esto era coherente con la definición de la petición como un acto de habla que supone un 'alto grado de opcionalidad' para el oyente (cortesía), comparado con el 'mínimo grado de opcionalidad' de la orden. Encontramos, en efecto, que las variaciones en el grado de expresión de este mismo componente se asocian con variaciones en los índices de prototipicidad de los enunciados. Concretamente, que mientras los índices de prototipicidad para orden disminuyen al bajar el grado en que los enunciados expresan el 'Deseo del hablante' -como en los ejemplos (20) y (21), comparados con (9)-, aumentan los índices de prototipicidad de los mismos para la categoría de la petición, quedando finalmente con índices muy cercanos. Asimismo, la disminución en el grado en que se expresa el conocimiento del hablante sobre la habilidad del oyente (ANTES 1) se asocia a los enunciados convencionalizados como en el ejemplo (8), mientras que la representatividad disminuye al eliminar parte de los recursos expresivos, como el formato interrogativo en (22):
(8) ¿Podrías cerrar la puerta?
(5,77 - petición / 2,05 - orden)
(9) Quiero que cierres la puerta.
(2,78 - petición / 5,00 - orden)
(20) Quisiera que cierres la puerta.
(4,26 - petición / 4,70 - orden)
(21) Querría que cierres la puerta.
(3,87 - petición / 3,30 - orden)
(22) Si pudieras cerrar la puerta.
(3,26 - petición / 2,79 - orden) 
Pérez Hernández y Ruiz de Mendoza (2002) consideran que este fenómeno supone la interacción de principios metonímicos con otras estructuras conceptuales, por ejemplo, con 'esquemas de distancia conceptual' (en el sentido de Taylor, 2003). Estos esquemas surgen de la conceptualización metafórica del 'tiempo' en términos de ‘espacio' ('esquema de distancia-proximidad'), e intervendrían en la conceptualización del nivel de implicación de los participantes en la comunicación (Pérez Hernández \& Ruiz de Mendoza, 2002).

Un 'esquema de distancia-proximidad' permite al hablante crear distintos grados de distancia entre ‘sí mismo' y su deseo de que $\mathrm{O}$ haga $\mathrm{A}$ (o entre ‘sí mismo' y el enunciado directivo que expresa ese deseo), logrando efectos de gradación en el componente metonimizado. Esto se condice con los resultados descritos. En el ejemplo (9), el uso del presente ('quiero que...') establece una distancia conceptual mínima entre el hablante y su deseo de que 0 haga $A$, mientras que el uso del subjuntivo y del condicional en (20) y (21), establecen una distancia mayor. La cortesía -o la 'opcionalidad' para al oyente- es menor en el primer caso (9) y mayor en (20) y (21).

\subsubsection{Componente NÚCLEO}

Con respecto al componente NÚCLEO, los resultados revelan que el aumento en el grado de expresión (enunciados aseverativos), como en los ejemplos (11) y (12), se asocia a un aumento de los índices de prototipicidad para orden. A la vez, la disminución se asocia a grados inferiores de prototipicidad de los enunciados para la misma categoría. Por ejemplo, mediante el uso del subjuntivo y del condicional, los enunciados (13) al (15) expresan el componente NúCLEO en menor medida que (11) y (12), obteniendo índices menores de prototipicidad para orden.

(11) Tienes que cerrar la puerta.

$(6,40$ - orden)

(12) Debes cerrar la puerta.

$(6,05$ - orden)

(13) Debieras cerrar la puerta

(4.65 - orden)

(14) Deberías cerrar la puerta

(4.40 - orden)

(15) Tendrías que cerrar la puerta

(4.40 - orden)

En otras palabras, el componente NÚCLEO no solo es central en las órdenes, sino que grados altos de expresión del mismo se asocian a índices también altos de prototipicidad para esta categoría. En este caso, la disminución en el grado de obligatoriedad de la acción del oyente no aumenta los índices de los enunciados hacia el polo categorial (opuesto) de la petición. Es probable que las variaciones en el grado de expresión del componente NÚCLEO en metonimias ilocutivas del tipo ejemplificado ('Deber + SV' o 'Tener que + SV') hagan oscilar la categorización entre el polo categorial de la orden y categorías intermedias como la sugerencia y el consejo, en las que sí está implicado cierto grado de obligatoriedad o necesidad de la acción para el oyente. 
Los enunciados aseverativos del tipo (11) y (12) crean una distancia conceptual mínima entre el oyente y su obligación de realizar la acción, mientras que desde los ejemplos (13) al (15) crean una distancia mayor. Así, la proximidad temporal conceptualiza mejor el compromiso del oyente con la acción, mientras que la distancia su disminución. Los enunciados (23) y (24) constituyen ejemplos adicionales de este juego de distancia-proximidad (proponemos interpretaciones ilocutivas plausibles en paréntesis):

(23) Tendrás que cerrar la puerta. (Orden).

(24) Tenías que cerrar la puerta. (Reproche, Queja).

\subsubsection{Componente DESPUÉS}

Los resultados obtenidos para este componente (ver Tablas 10 y 11) confirman también nuestra sub-hipótesis (a.2.). Los enunciados que expresan más categóricamente el componente DESPUÉS -central en órdenes, según la sub-hipótesis (a.1.)-, como en los ejemplos (16) y (17), obtuvieron los índices más elevados de prototipicidad para orden (Tabla 11). El enunciado (18), un caso de la metonimia ilocutiva ACCIÓN PRESENTE POR ACCIÓN FUTURA, obtuvo también un índice importante para orden. Los índices bajan, en cambio, al disminuir el grado de manifestación de este mismo componente en el resto de los enunciados (ver Tabla 11), hasta llegar al enunciado (19), con el índice mínimo:

(16) Tú cerrarás la puerta

(17) Vas a cerrar la puerta

(18) Tú cierras la puerta, ¿ya?

(19) ¿Cerrarías la puerta?
(2,22 - petición / 5,95 - orden)

(1,91 - petición / 5,40 - orden)

(3,26 - petición / 5,05 - orden)

(5,57 - petición / 1,75 - orden)

Desde el punto de vista de la petición (Tabla 10), este mismo enunciado obtuvo el índice de prototipicidad más significativo del grupo $(5,57)$. También en este caso se confirma nuestra sub-hipótesis (a.2.), puesto que el formato interrogativo y condicional de (19), comparado con el modo declarativo de (16) y (17), aumenta la virtualidad de la acción futura del oyente, disminuyendo el grado de manifestación del componente ilocutivo DESPUÉS. Con el uso del condicional, en (19), se establece, nuevamente, mayor 'distancia conceptual' entre el hablante y su objetivo de que el oyente haga A ('O hará A') y entre el oyente y la acción futura. Mientras que con el modo interrogativo se deja al oyente la posibilidad de negarse a realizar la acción, lo que implica mayor grado de 'opcionalidad'.

Pero, como señalamos en la sección 2.2.1.3., el elevado índice para petición obtenido por el 
enunciado (19), pone en duda nuestra conclusión de una baja probabilidad de la metonimia ilocutiva ACCIÓN FUTURA DEL OYENTE POR PETICIÓN.

Creemos que el análisis recién hecho, acerca de la relación entre el formato condicional interrogativo de (19) y el efecto de gradación del componente representado (DESPUÉS), permite explicar la prototipicidad de este enunciado sin anular completamente nuestra predicción. Cuando ciertos componentes del escenario de directivos son centrales para una categoría directiva específica (para la orden, por ejemplo), se comportan de tal manera que, el aumento en su grado de expresión supone, también, el aumento del potencial ilocutivo (prototipicidad), para esa categoría, de los enunciados que son funciones metonímicas de esos componentes; mientras que la disminución disminuye ese potencial. Según esto, como el componente DESPUÉS es central en la evocación metonímica del $\mathrm{MCl}$ de la orden, los enunciados que destacan este componente requieren marcadores pragmáticos o semánticos que disminuyan su grado de expresión, para tener interpretaciones peticionarias. Tenemos así otro caso en el que un modelo metonímico interactúa con un esquema basado en una metáfora: esquema de distancia-proximidad.

\section{CONCLUSIONES}

Nuestras pruebas pretendían medir la potencialidad de un grupo de enunciados para la expresión de la fuerza directiva de orden o de petición con independencia de un contexto concreto de enunciación. Para ello activamos el modelo correspondiente solicitando a los sujetos que evaluaran los enunciados en relación con la categoría específica: petición (Prueba A) u orden (Prueba B). Nuestros resultados proporcionarían evidencia de la utilidad o validez de la perspectiva metonímica basada en la existencia de MCls escénicos para dar cuenta de la riqueza expresiva ilocutiva indirecta. La propuesta sobre MCls de Lakoff (1987), bajo la forma desarrollada por Pérez Hernández y Ruiz de Mendoza (2002), ofrecen, según esto, no solo la posibilidad de identificar formatos potencialmente útiles para expresar una determinada ilocución, sino la de proponer un límite relativo de adecuación de esa gran riqueza y variabilidad.

Los resultados de ambas pruebas de prototipicidad han proporcionado evidencia en apoyo de la hipótesis (a) de Thornburg y Panther (1997), según la cual es posible predecir la probabilidad de que un componente del escenario de directivos esté en una relación metonímica con el escenario mismo. Sin embargo, esta hipótesis no nos dice mucho sobre la producción (e interpretación) de actos de habla directivos específicos, es decir, no nos dice qué componentes ni, por lo tanto, qué operaciones metonímicas son centrales en la producción y en la percepción del potencial ilocutivo de enunciados no literales o no convencionalizados, con respecto a subtipos de actos de habla directivos. Nuestras pruebas proporcionan datos útiles para respaldar propuestas sobre este punto. Principalmente, ofrecen evidencia de la centralidad de los componentes del 
escenario de directivos de Thornburg y Panther en la expresión metonímica de órdenes y peticiones (sub-hipótesis (a.1.)), y de aspectos que el enfoque metonímico no considera, como la diferencias en representatividad de los enunciados que expresan, en diversos grados, un mismo componente (sub-hipótesis (a.2)). La participación de esquemas conceptuales en la expresividad ilocutiva indirecta ( $\mathrm{y}$ en la interpretación de la fuerza ilocutiva indirecta) de enunciados del español chileno es una idea que puede desarrollarse considerando ejemplos cuya potencialidad ilocutiva ha sido testada en hablantes.

Particularmente, hemos encontrado que no todos los componentes del escenario general de directivos de Thornburg y Panther son centrales para todos los subtipos de esta clase de actos de habla. Así, las metonimias de los componentes ANTES 2 ('Motivación/deseo de $\mathrm{H}$ de que $\mathrm{O}$ haga A'), NÚCLEO ('O está bajo la obligación de hacer A') y DESPUÉS ('O hará A') son altamente útiles en la realización de directivos fuertes (de 'alta obligatoriedad' y 'baja opcionalidad'), como las órdenes; mientras que las metonimias del componente ANTES 1 ('Habilidad de 0 para hacer $A^{\prime}$ ), lo son para la realización de directivos menos fuertes, como las peticiones. Sin embargo, en todos estos casos los diferentes puntos en la escala de prototipicidad alcanzada dependieron de variaciones en el grado de manifestación de los componentes del escenario de directivos, asociadas a efectos de gradación en la manifestación de propiedades específicas (opcionalidad, cortesía). Por ello, nuestros datos apoyan la sub-hipótesis (a.1) solo si se considera la centralidad de un componente y, por tanto, la probabilidad de la metonimia correspondiente, en relación con el grado en que ese componente se encuentre representado en el $\mathrm{MCl}$ de Actos de Habla Directivos. Los componentes ANTES 2 y NÚCLEO son centrales para órdenes porque en el $\mathrm{MCl}$ de Directivos se representan en su grado óptimo. Entonces, un enunciado que exprese el componente en este grado ideal, aun cuando no represente un formato convencionalizado para la expresión de órdenes, como en (12), producirá una alta percepción de pertenencia directiva (será un caso representativo de directivo fuerte), la que disminuirá si su expresión es menor, como en (14). Teóricamente, el ejemplo (12) requiere una marca contextual mayor que un imperativo como (25) -que instancia una 'construcción ilocutiva'- para que pueda recibir esa interpretación.

(12) Debes cerrar la puerta.

(6,05 - orden)

(14) Deberías cerrar la puerta

(4.40 - orden)

(25) Cierra la puerta.

Pero, si el contexto específico se marca, activando el $\mathrm{MCl}$ de orden, como en la Prueba B, la referencia metonímica funciona, y existe también una alta probabilidad, como vimos más atrás, de que encontremos diferencias en el potencial ilocutivo directivo de enunciados metonímicos de NÚCLEO que expresan diversos grados de obligatoriedad, como en (15) y (23). 
El componente ANTES 1 ('Habilidad del oyente'), por otro lado, ha mostrado ser central para peticiones, pero siempre que el enunciado metonímico lo exprese en un nivel mínimo, pues, dado que en el modelo para directivos el reconocimiento de la habilidad del oyente se representa en su grado óptimo, el aumento de la opcionalidad (clave en $\mathrm{MCl}$ específico de petición) dependerá de que la certeza acerca de esa habilidad disminuya. De ahí que los casos prototípicos de petición coincidan con 'preguntas' por la habilidad del oyente, como en (7) y (8), que representan peticiones directas o convencionales en español (Ruiz de Mendoza, 2001). Esto es coherente con nuestra intuición como hablantes de que los enunciados en que la habilidad del oyente se expresa más categóricamente (por ejemplo, 'Tú puedes cerrar la puerta' y 'Puedes cerrar la puerta') no califican como buenos ejemplos de la categoría de la petición, pero sí de categorías directivas más fuertes (como 'orden' y 'dar permiso', por ejemplo). Los actos de habla directivos de la orden y de la petición corresponden así a dos modos diferentes de manifestación de un modelo complejo más general de directividad ilocutiva. Los casos prototípicos corresponden a su vez a contextos que expresan en un grado particular o prototípico cada una de las propiedades y componentes escénicos asociados a ese modelo general. En este caso, nuestras pruebas apoyan las conclusiones de Pérez Hernández (2000).

Asimismo, la centralidad del componente ANTES 2 ('Deseo de $\mathrm{H}$ de que $\mathrm{O}$ haga A') en directivos fuertes como la orden, explica la necesidad de utilizar recursos que aminoren la fuerza directiva para expresar metonímicamente actos directivos menos impositivos. Vimos, por ejemplo, que (9) es altamente representativo de orden (índice de 5.00), de manera que su uso para expresar peticiones precisa la introducción de marcadores peticionarios como en (26). Esto es coherente con el aumento de los índices de prototipicidad para petición de los enunciados (20) y (21), que expresan menor grado de ANTES 2 ('Deseo de $\mathrm{H}$ de que $\mathrm{O}$ haga $\mathrm{A}^{\prime}$ ):

(9) Quiero que cierres la puerta.

(2,78 - petición / 5,00 - orden)

(26) Quiero que cierres la puerta, por favor.

(20) Quisiera que cierres la puerta.

(4,26 - petición / 4,70 - orden)

(21) Querría que cierres la puerta.

(3,87 - petición / 3,30 - orden)

Finalmente, nuestros datos acerca de la percepción del potencial ilocutivo para órdenes y peticiones de las metonimias de DESPUÉS, nos permiten relacionar el efecto de gradación con el uso de mecanismos que aumentan o aminoran el grado de certeza del hablante acerca de la realización futura de la acción por el oyente. Así, los enunciados formulados en futuro simple (16) o en presente (18), crean la impresión de que el hablante tiene mayor seguridad acerca de la realización de la acción, comparados con enunciados interrogativo-condicionales como en (19).

Pérez Hernández y Ruiz de Mendoza (2002) proponen, como señalamos, que los efectos de gra- 
dación de los componentes ilocutivos (así como la disminución o aumento en el número de estos) que expresa un enunciado, son el resultado de la interacción de modelos cognitivos 'proposicionales' (como los escenarios de actos de habla), 'metonímicos', 'metafóricos' y de 'esquemas de imagen'. Ejemplos de la interacción de modelos metafóricos son los 'esquemas de distancia conceptual', que producen gradación en el componente DESPUÉS del escenario de directivos. Nuestras pruebas y análisis proporcionan datos del español de Chile en apoyo de la idea de que el 'potencial construccional ilocutivo' de los enunciados depende de esta clase de efectos, basados en la interacción de principios cognitivos. Un $\mathrm{MCl}$ de Acto de Habla representa el sustrato unificador de ciertos contextos ilocutivos y la base para las operaciones que, reflejadas en los enunciados, guían y explican la percepción de su potencial pragmático. Mediante la obtención de los índices de prototipicidad ha sido posible determinar, con cierto grado de certeza, no solo la probabilidad de que ciertos enunciados sean interpretados como peticiones y órdenes, y usados para realizarlas, sino, principalmente, ofrecer datos que apoyen con ejemplos testados las propuestas de la pragmática de los actos de habla para dar cuenta de la variabilidad expresiva y de sus límites, en el contexto de la lingüística cognitiva.

\section{REFERENCIAS BIBLIOGRÁFICAS}

Berlin, B. \& Kay, P. (1969). Basic color terms: Their universality and evolution. Berkeley: University of California Press.

Coleman, L. \& Kay, P. (1981). Prototype semantics: The English word lie. Language, 57(1), 26-44.

Fauconnier, G. (1985). Mental spaces. Cambridge, Mass.: MIT Press.

Fillmore, Ch. (1982). Frame semantics. En Linguistic Society of Korea (Eds.), Linguistics in the morning calm (pp. 111-138). Seoul: Hanshin.

Grice, P. (1975). Logic and conversation. En P. Cole \& J. Morgan (Eds.), Syntax and semantics (pp. 41-58). New York: Academic Press.

Johnson, M. (1987). The body in the mind: The bodily basis of meaning, imagination, and reason. Chicago: University of Chicago Press.

Lakoff, G. (1987). Women, fire, and dangerous things. What categories reveal about the mind. Chicago, London: The University of Chicago Press.

Lakoff, G. \& Johnson, M. (1995). Metáforas de la vida cotidiana. Madrid: Cátedra.

Labov, W. (1973). The boundaries of words and their meanings. En C. Bailey \& R. Shuy (Eds.), New ways of analysing variation in English (pp. 340-373). Washington: Georgetown University Press.

Langacker, R. (1986). Foundations of cognitive grammar. Stanford: University Press. 
Leech, G. (1997). Principios de pragmática, Universidad de la Rioja: Servicio de Publicaciones.

Panther, K. \& Thornburg, L. (1997). A cognitive approach to inferencing in conversation. Journal of Pragmatics, 30, 755-769.

Panther, K. \& Thornburg, L. (1999). The potentiality for actuality metonymy in English and Hungarian. En K. Panther \& G. Radden (Eds.), Metonymy in language and thought (pp. 333-357). Amsterdam and Philadelphia: Benjamins.

Panther, K. \& Thornburg, L. (2007). Metonymy. En D. Geeraerts \& H. Cuyckens (Eds.), The Oxford Handbook of Cognitive Linguistics (pp. 236-263). Oxford: University Press.

Pérez Hernández, L. (2000). Cómo conseguir que la gente obedezca tus palabras: Un análisis cognoscitivo de las órdenes en español. Revista Española de Lingüística Aplicada, Volumen Monográfico: Estudios Cognoscitivos del Español, 375-393.

Pérez Hernández, L. \& Ruiz de Mendoza, F. (2002). Grounding, semantic motivation, and conceptual interaction in indirect directive speech acts. Journal of Pragmatics, 34, 259-284.

Rivano, E. (1999). Un modelo para la descripción y análisis de la metáfora. Logos, 9, 41-51.

Rosch, E. (1973a). Natural categories. Cognitive Psychology, 4, 328-350.

Rosch, E. (1973b). On the internal structure of perceptual and semantic categories. En T. Moore (Ed.), Cognitive development and the acquisition of language (pp. 111-144). New York: Academic Press.

Rosch, E. (1975). Principles of categorization. En E. Rosch \& B. Lloyd (Eds.), Cognition and categorization (pp. 27-48). Hillsdale: Erlbaum.

Rosch, E. \& Mervis, C. (1975). Family resemblances: Studies in the internal structure of categories. Cognitive Psychology, 7, 573-605.

Ruiz de Mendoza, F. (2001). Lingüística cognitiva: Semántica, pragmática y construcciones, Revista electrónica clac, 8 [en línea]. Disponible en: http://www.ucm.es/info/circulo/ no8/ruiz.htm

Rumelhart, D. (1975). Notes on a schema for stories. En D. Bobrow \& A. Collins (Eds.), Representation and understanding: Studies in cognitive science (pp. 211-236). New York: Academic Press.

Schank, R. \& Abelson, R. (1977). Scripts, plans, goals, and understanding. Hillsdale, N.J.: Lawrence Erlbaum.

Searle, J. (1969). Speech acts. An essay in the philosophy of language. Cambridge: University Press.

Searle, J. (1975). Indirect speech acts. En P. Cole \& J. Morgan (Eds.), Syntax and semantics (pp. 59-82). New York: Academic Press.

Sperber, D. \& Wilson, D. (1994). La relevancia. Comunicación y procesos cognitivos. Madrid: Visor. 
Taylor, J. (2003). Linguistic categorization. New York: Oxford University Press.

Thornburg, L. \& Panther, K. (1997). Speech act metonymies. En W. Liebert, G. Redeker \& L. Waugh (Eds.), Discourse and perspectives in cognitive linguistics (pp. 205-219). Amsterdam and Philadelphia: Benjamins.

Wittgenstein, L. (1953). Philosophical investigations. New York: MacMillan. 